\title{
Perfil epidemiológico de la oclusión dental en escolares de Envigado, Colombia
}

\section{Epidemiological profile of dental occlusion in children attending school in Envigado, Colombia}

\author{
Paola A. Urrego-Burbano ${ }^{1}$, Lina P. Jiménez-Arroyave ${ }^{1}$, Miguel Á. Londoño-Bolívar ${ }^{1}$, \\ Mario Zapata- Tamayo ${ }^{2}$ y Paola Botero-Mariaca ${ }^{1}$ \\ 1 Universidad Cooperativa de Colombia, Sede Medellín. Colombia. pub89ortodoncia@gmail.com, \\ ljimenezortodoncia@gmail.com, mianlobo78@gmail.com, preciado@une.net.co \\ 2 Universidad de Antioquia. Medellín, Colombia.mzapata@udea.edu.co
}

Recibido 3 Marzo 2011/Enviado para Modificación 5 Diciembre 2011/Aceptado 19 Diciembre 2011

\section{RESUMEN}

Objetivos La oclusión ideal es un estándar hipotético basado en las relaciones morfológicas de los dientes, se caracteriza por una perfección en la anatomía y posición dental, contactos mesiodistales, alineamiento en el arco e interdigitación dental. El propósito de este estudio fue determinar el perfil epidemiológico de la oclusión dental en escolares del municipio de envigado de instituciones públicas, del área urbana y rural, durante el primer semestre de 2010.

Materiales y Métodos Se realizo un estudio epidemiológico descriptivo de corte transversal en pacientes con edades entre los 5 y los 12 años de edad. Se calculo un tamaño de muestra de 436 estudiantes, distribuidos en 6 instituciones educativas del área rural y 6 del área urbana. Se realizó un examen clínico de la cavidad oral donde se evaluaron diferentes variables oclusales en los tres planos del espacio: sagital, trasversal y vertical.

Resultados Se evaluaron un total 436 sujetos, con una edad promedio de 8 años $(8 \pm 1,9)$, la maloclusión con mayor prevalencia para la dentición permanente, fue del 49,7\% (176/354) para la maloclusión clase I, 43,5\% (154/354) para la maloclusión clase II y del 6,8 \% (24/354) para la maloclusión clase III.

Conclusión La maloclusión clase I de Angle fue la más prevalente con alteraciones en los planos vertical y trasversal y problemas de espacio en el segmento anterior.

Palabras Clave: Maloclusión de Angle clase I, Maloclusión de Angle clase II, Maloclusión de Angle clase III, prevalencia (fuente: DeCS, BIREME).

\section{ABSTRACT}

Objectives Ideal occlusion is a hypothetical standard based on teeth's morphological relationships and is characterised by perfection in anatomy and dental position, mesiodistal contacts, arch alignment and dental interdigitation. This investigation 
was aimed at determining the epidemiological profile of dental occlusion of children attending urban and rural public schools in Envigado during the first semester, 2010.

Materials and Methods A cross-sectional, prospective, descriptive epidemiological study was carried out on patients ranging from 5 to 12 years of age. A sample size of 436 students from 6 rural schools 6 urban institutions was calculated. The oral cavity was clinically examined and different occlusal variables on the three planes of space were evaluated: sagittal, transversal, and vertical.

Results 436 subjects were evaluated; average age was 8 years $( \pm 1.9)$. The most prevalent malocclusion for permanent dentition was Angle's Class I $(49.7 \%$, $176 / 354)$, followed by Angle's Class II $(43.5 \%, 154 / 354)$ and Angle's Class III $(6.8 \%$, 24/354).

Conclusion Angle's Class I malocclusion was the most prevalent condition with alterations in both vertical and transversal planes and space problems in the anterior segment.

Key Words: Angle's class I malocclusion, Angle's class II malocclusion, Angle's class III malocclusion, prevalence (source: MeSH, NLM).

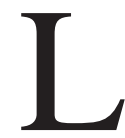

a oclusión de los dientes involucra su alineación e intercuspidación e influye en diversas funciones, la estética facial y la salud del sistema estomatognático. Las maloclusiones definidas como desviaciones de organizaciones dentales ocurren durante el desarrollo craneofacial; y su clasificación inicial fue descrita por Edward Angle ${ }^{(1)}$, quien se basó en la relación antero posterior del primer molar, cuya mala oclusión influye en las relaciones esqueléticas. Las alteraciones pueden ocurrir en el plano vertical, sagital o transversal (1-4). La oclusión ideal es un estándar hipotético basado en las relaciones morfológicas de los dientes, se caracteriza por una perfección en la anatomía y posición dental, contactos mesiodistales, alineamiento en el arco e interdigitación dental (2). Lo ideal es difícil de encontrar y es considerada una norma teórica más que una condición normal. La etiología de la maloclusión es multifactorial donde factores genéticos y medio ambientales pueden influir (3). Según la Organización Mundial de la Salud (OMS), las maloclusiones ocupan el tercer lugar de prevalencia dentro de las patologías en Salud bucodental, luego de la caries dental y de la enfermedad periodontal (1). En Latinoamérica según datos de la Organización Panamericana de la Salud OPS, existen altos niveles de incidencia y prevalencia de maloclusiones que superan el $80 \%$ de la población (1-3) siendo uno de los motivos de consulta mas frecuentes en las clínicas dentales (2). 
Se han realizado diversos estudios para tratar de describir la distribución de la maloclusión, encontrando una prevalencia entre 39 y 93 \%, debido a diferentes métodos de recolección de la información, diferente muestra y razas distintas (3). En Colombia se encuentran varios estudios importantes, como el realizado en Bogotá por Thilander y col (7), en Medellín en el 2002 (4) y el ENSAB (5) que considero aspectos distintos a los otros. Determinar la prevalencia de las maloclusiones es importante para establecer lineamientos en las políticas preventivas y de tratamiento en una comunidad; por lo tanto este estudio tiene como objetivo determinar la prevalencia de la maloclusión en escolares de 5 a 12 años de edad en instituciones públicas urbanas y rurales del Municipio de Envigado.

\section{MATERIALES Y MÉTODOS}

Se realizo un estudio epidemiológico descriptivo de corte transversal, en escolares de colegios públicos (urbanos y rurales) del municipio de Envigado. El diseño muestral tuvo como base un muestreo probabilístico (debido a que no se conocían las características oclusales de la población a observar) georeferenciado, estratificado proporcionalmente por ubicación geográfica de la institución educativa y pareado por genero. La muestra fue calculada con Epi-Info versión 6.04 con un nivel de confiabilidad del $95 \%$, estimación de error $5 \%$ y probabilidad del $50 \%$, el universo muestral fue de 8279 estudiantes de 30 instituciones de educación primaria públicas, de las cuales 20 estaban ubicadas en el área urbana y 10 en zona rural del municipio de Envigado (Antioquia); el cálculo final fue de 376 estudiantes ajustado por estratificación a 436 estudiantes, distribuidos en 6 instituciones educativas del área rural y 6 del área urbana seleccionados aleatoriamente. Los estudiantes de cada institución fueron seleccionados al azar según los siguientes criterios de inclusión: escolares matriculados en colegios públicos del municipio de Envigado (rurales y urbanos) en el primer semestre del 2010, y que se encontraran en una edad entre 5 y 12 años; no se incluyeron estudiantes con presencia de algún síndrome mental o físico, con presencia de enfermedades sistémicas que afectaran el desarrollo esquelético normal, que hayan o estén recibiendo tratamiento ortodóncico interceptivo, escolares de difícil manejo que no permitan la exploración de la cavidad oral y consentimiento informado no firmado. La recolección de la información fue realizada por tres examinadores, los cuales para asegurar confiabilidad de la información fueron estandarizados 
por un experto en el área (ortodoncista con investigaciones previas en el área (4)), dejándolos en capacidad técnica adecuada para realizar las observaciones. Posteriormente los tres examinadores realizaron una prueba piloto con una muestra equivalente al $10 \%$ de la muestra del estudio, la cual fue valorada en variables cualitativas utilizando el índice de Kappa de Kohen y en variables cuantitativas se utilizó el Coeficiente de Correlación de Pearson; mostrando un índice de Kappa interexaminador de 72,3 \% (IC95\% = 53,0 \% - 91,0\%), índice de Kappa intraexaminador de 78,33 $\%$ (IC95\% = 67,13 \% - 86,5 \%), un Coeficiente de Correlación de Pearson interexaminador de $\mathrm{R}^{2}=0,765$ y un promedio intraexaminador de $\mathrm{R}^{2}=0,787$. Los datos obtenidos fueron analizados utilizando el paquete estadístico para las Ciencias Sociales para Windows (SPSS Inc., Chicago, Illinois, EE.UU. Vers.18). Para determinar diferencias en la necesidad de tratamiento entre los subgrupos a comparar, y para identificar categorías de riesgo por asociación se utilizo la Razón de Disparidad (RD), cada medida con su correspondiente intervalo de confianza (I.C.95\%); el nivel de significancia se fijó en 0,05. Según la resolución 8430 de 1993, la investigación fue clasificada como riesgo mínimo y se diseño un consentimiento informado para los padres. El proyecto fue aprobado por el comité de ética de la universidad y por la secretaria de salud y educación de Envigado. El formato de recolección de la información presentaba variables sociodemográficas como: edad, sexo y ubicación geográfica. El estadío de la dentición se determinó usando las etapas del desarrollo dental descritas por Bjork en 1964 (7). Se evaluó el plano sagital (relación molar decidua y permanente, relación canina y overjet), el plano transversal (mordidas cruzadas, tijera y línea media) y en el plano vertical (overbite). Dentalmente se valoró la presencia o ausencia de apiñamiento.

\section{RESULTADOS}

En el estudio se evaluaron un total 436 sujetos, con una edad promedio de 8 años (D.E 1,9), con una edad mínima de 5 años y una edad máxima de 12 años, el 61 \% (266/436) de la muestra se encuentra en el rango de edad de 7 a 10 años. La distribución correspondiente a la ubicación geográfica de las instituciones educativas se presento en un mayor porcentaje para la zona urbana con un 51,8 \% (226/436). Para el tipo de dentición se presenta una tendencia invertida de los porcentajes de frecuencia desde la dentición mixta hasta la dentición permanente decidua, con frecuencias que van desde 34,9 \% (152/436) hasta 7,8 \% (34/436) respectivamente. 
Cuando se evaluó la prevalencia de la maloclusión para la dentición permanente (178 escolares), se encontró el 49,7 \% (176/354) de maloclusión clase I, 43,5 \% (154/354) de maloclusión clase II y del 6,8 \% (24/354) de maloclusión clase III. Con respecto a la dentición decidua, (82 escolares), se encontró un $32.9 \%$ (27/82) con plano terminal recto, un 57.3 \% (47/82) con escalón mesial y un 9,8 \% (8/82) con escalón distal. La mayor frecuencia de maloclusión en dentición permanente se presentó en el grupo etareó de los 9 a 10 años con un 50,4 \% (68/135), seguido del rango de edad de los 7 a los 8 años con una frecuencia relativa de 48,1\% (63/131); la mayor frecuencia de escolares en los que no se pudo clasificar la maloclusión se presento en el grupo etareó de los 5 a 6 años con un 66.1 $\%(74 / 112)$.

Cuando se valoraron las diferencias en las proporciones de caracterización de la muestra en el grupo con maloclusión por variable socio demográfica evaluada, se encontró que sí existían diferencias estadísticamente significativas en edad, y tipo de dentición con un valor de $p$ menor 0,005 y un $\mathrm{Z}$ del $95 \%$ (Tabla 1 ). Al analizar las variables en los tres planos del espacio en individuos con maloclusión en dentición permanente, se encontró en el plano sagital una relación molar clase I, acompañada con mayor frecuencia de una canina clase II en un 10,8 \% (19/176), la relación molar clase II acompañada de una relación canina clase II en un 16,9 \% (26/154); y finalmente la relación molar clase III con una relación canina clase III en un 8,3 \% (4/24) (Tabla 2). Al relacionar el tipo de maloclusión con la magnitud del overjet se encontró que para la maloclusión clase I había una frecuencia mayor de overjet adecuado (1-3mm) en un 70,4 \% (124/176) (Figura 1). En el total de la muestra el overjet se presentó de manera adecuada en un 68,6 \% (299/436), aumentado en un 23,4\% (102/436), invertido en un 2,8 \% (12/436) y no se pudo determinar por ausencia de incisivos en un 5,3\% (23/436). Al hacer el cruce de variables entre el tipo de maloclusión y overbite se encontró similar para los tres tipos de maloclusión (Figura 2).

Cuando se valoró solo el overbite se encontró un adecuado, del 48,6\% (212/436), seguido de una mordida profunda del 39,2\% (171/436), abierta en un 3,0 \% (13/436) y una mordida borde a borde en un 3,9 \% (17/436). Finalmente en el plano trasversal se encontró una mordida cruzada completa en un 11,0 \% (48/436), presentándose en un 10,6 \% (46/436) de manera 
unilateral y bilateral en un 3,7 \% (16/436). Con respecto a la línea media dental se observó coincidencia en un 69,3 \% (302/436) y no coincidencia en un 30,7 \% (134/436).

Tabla 1. Características de la muestra según tipos de maloclusión permanente y decidua

\begin{tabular}{|c|c|c|c|c|c|c|c|}
\hline \multirow[t]{2}{*}{ Característica } & \multicolumn{2}{|c|}{$\begin{array}{l}\text { Maloclusión } \\
\text { Tipo I y III } \\
(\mathrm{N}=178)\end{array}$} & \multicolumn{2}{|c|}{$\begin{array}{l}\text { Maloclusión } \\
\text { tipo I (N=176) }\end{array}$} & \multicolumn{2}{|c|}{$\begin{array}{l}\text { Maloclusión } \\
\text { dentición } \\
\text { decidua } \\
(\mathrm{N}=82)\end{array}$} & \multirow[t]{2}{*}{ Valor $\mathrm{P}^{*}$} \\
\hline & $\mathrm{N}$ & $\%$ & $\mathrm{~N}$ & $\%$ & $\mathrm{~N}$ & & \\
\hline $\begin{array}{l}\text { Edad en años } \\
\text { (media/desviación) }\end{array}$ & & $(5,7 / 0,8)$ & & $(8,6 / 1,7)$ & & $(8.7 / 1.7)$ & $<0.001^{\star *}$ \\
\hline $5-6$ & 20 & 17,9 & 18 & 16,1 & 74 & 66,1 & 0,000 \\
\hline $7-8$ & 63 & 48,1 & 61 & 46,6 & 7 & 5,3 & \\
\hline $9-10$ & 68 & 50,4 & 66 & 48,9 & 1 & 0,7 & \\
\hline $11-12$ & 27 & 46,6 & 31 & 53,4 & 0 & 0,0 & \\
\hline \multicolumn{8}{|l|}{ Zona } \\
\hline Rural & 88 & 41,9 & 88 & 41,9 & 34 & 16,2 & 0,401 \\
\hline Urbana & 90 & 39,8 & 88 & 38,9 & 48 & 21,2 & \\
\hline \multicolumn{8}{|l|}{ Tipo de dentición } \\
\hline Inicial & 57 & 37,5 & 58 & 38,2 & 37 & 24,3 & 0,000 \\
\hline $\begin{array}{l}\text { Mixta } \\
\text { intertransicional }\end{array}$ & 64 & 53,8 & 54 & 45,4 & 1 & 0,8 & \\
\hline Mixta final & 40 & 45,5 & 47 & 53,4 & 1 & 1,1 & \\
\hline \multirow[t]{2}{*}{ Permanente } & 17 & 50,0 & 17 & 50,0 & 0 & 0,0 & \\
\hline & 0 & 0,0 & 0 & 0,0 & 43 & 43 & \\
\hline
\end{tabular}

*Prueba Chi-cuadrado; **Prueba Kruskal-Wallis

Figura 1. Relación molar vs. overjet

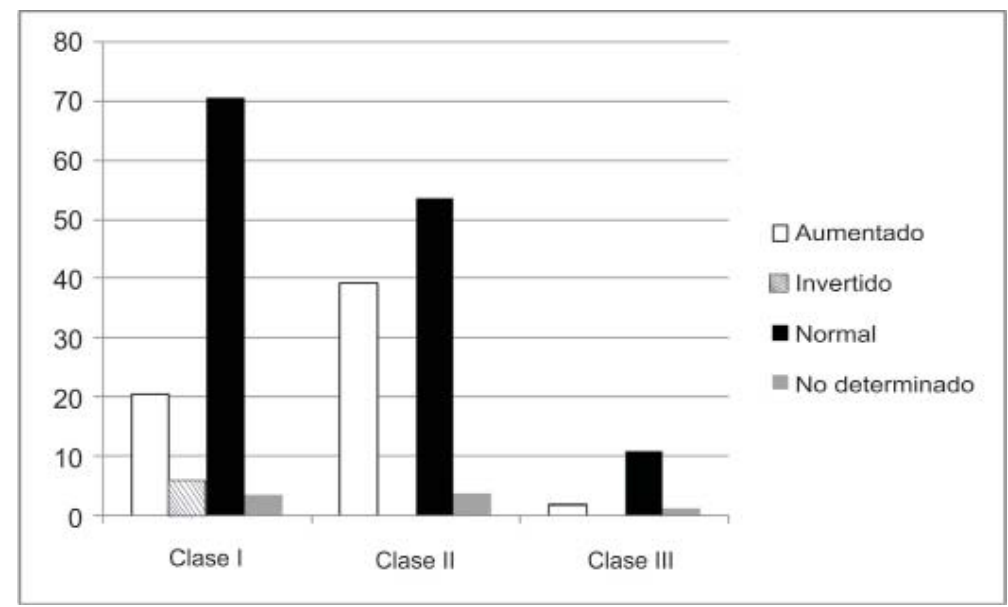


Al evaluar apiñamiento se observó un 57,8 \% (252/436) de frecuencia, presentándose en un 58,3 \% (254/436) en la zona anterior del arco únicamente sin poder determinarse en un $41,7 \%(182 / 436)$ por ausencia de incisivos y no existió apiñamiento en un 42,2 \% (184/436); Cuando se evaluó el posible nivel de relación entre el apiñamiento y la edad, se observó que el grado de asociación tiene una tendencia de aumento progresivo con el aumento de edad cronológica , presentándose un mayor valor de asociación en el estrato de edad de los 11-12 años con un OR de 3.1 (Tabla 3).

Tabla 2. Comparación de tipos de maloclusión permanente y decidua

\begin{tabular}{|c|c|c|c|c|c|c|c|}
\hline \multirow[t]{2}{*}{ Característica } & $\begin{array}{l}\text { Malc } \\
\text { y II }\end{array}$ & $\begin{array}{l}\text { lusión I } \\
\text { J=178) }\end{array}$ & \multirow{2}{*}{\multicolumn{2}{|c|}{$\begin{array}{l}\text { Maloclusión tipo } \\
\text { I / maloclusión } \\
\text { dentición decidua } \\
(\mathrm{N}=258)\end{array}$}} & \multirow[t]{2}{*}{ OR } & \multirow[t]{2}{*}{$\begin{array}{c}\text { IC95 } \\
\%\end{array}$} & \multirow[t]{2}{*}{ Valor $\mathrm{p}^{\star}$} \\
\hline & $\mathrm{n}$ & $\%$ & & & & & \\
\hline $\begin{array}{l}\text { Edad en años } \\
\text { (media/desviación) }\end{array}$ & & $(8,6 / 1,7)$ & & $(7,8 / 2,0)$ & & & $<0,001^{*}$ \\
\hline $5-6$ & 20 & 11,2 & 92 & 35,7 & 1,0 & & * 0,000 \\
\hline $7-8$ & 63 & 35,4 & 68 & 26,4 & 4,3 & $2,4-7,7$ & \\
\hline $9-10$ & 68 & 38,2 & 67 & 26,0 & 4,7 & $2,6-8-4$ & \\
\hline $11-12$ & 27 & 15,2 & 31 & 12,0 & 4,0 & $2,0-8,1$ & \\
\hline \multicolumn{8}{|l|}{ Zona } \\
\hline Rural & 88 & 49,4 & 122 & 47,3 & 1,0 & & 0,659 \\
\hline Urbana & 90 & 50,6 & 136 & 52,7 & 0,9 & $0,6-1,3$ & \\
\hline \multicolumn{8}{|l|}{ Tipo de dentición } \\
\hline Inicial & 57 & 32,0 & 95 & 36,8 & 1,0 & & 0,000 \\
\hline $\begin{array}{l}\text { Mixta } \\
\text { intertransicional }\end{array}$ & 64 & 36,0 & 55 & 21,3 & 1,9 & $1,2-3,2$ & \\
\hline Mixta final & 40 & 22,5 & 48 & 18,6 & 1,4 & $0,8-2,4$ & \\
\hline Permanente & 17 & 9,6 & 17 & 6,6 & 1,7 & $0,8-3,5$ & \\
\hline $\begin{array}{l}\text { Dentición } \\
\text { decidua }\end{array}$ & 0 & 0,0 & 43 & 16,7 & & & \\
\hline
\end{tabular}

Figura 2. Relación molar vs. overbite

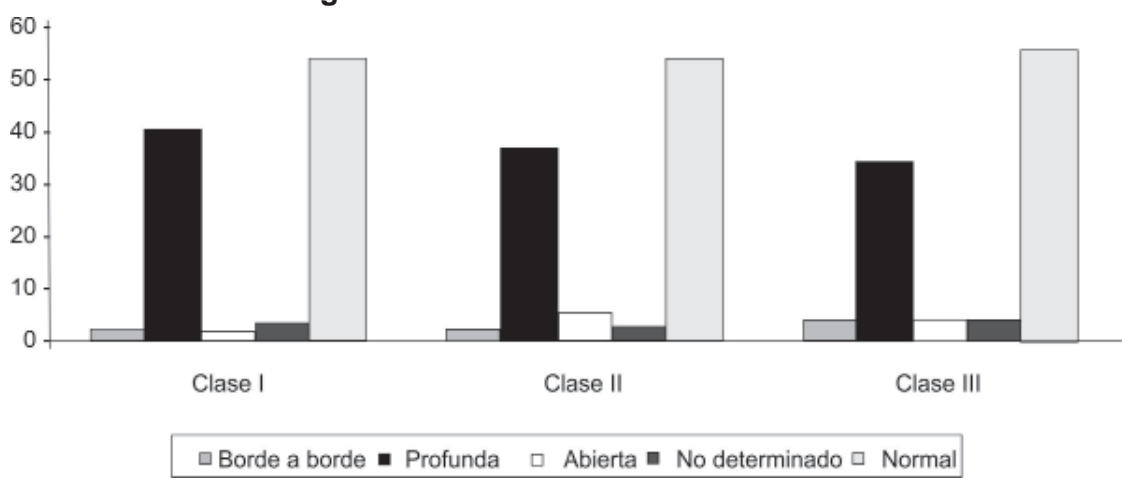


Por otra parte, el espaciamiento se presentó en un 66,7 \% (291/436) ubicado sólo en la zona anterior y no se pudo cuantificar por ausencia de incisivos en un 33,3 \% (145/436).cuando se evaluó el nivel de relación existente entre espaciamiento y los grupos de edad evaluados se encontró que existe una diferencia estadísticamente significativa $(\mathrm{p}<0,05)$, pero al evaluar la asociación utilizando la $\mathrm{RD}$ se encuentra que ninguna de ellas es estadísticamente significativa; esta misma situación se presento al evaluar tipo de dentición con respecto al espaciamiento; el valor de $\mathrm{p}(\mathrm{p}<0,05)$ sigue mostrando diferencias estadísticamente significativas entre los 5 tipos de dentición, pero el valor de la RD no muestra nivel de asociación estadísticamente significativa (Tabla 4).

Tabla 3. Características de los individuos según el apiñamiento

\begin{tabular}{|c|c|c|c|c|c|c|c|}
\hline \multirow{2}{*}{ Característica } & \multirow{2}{*}{\multicolumn{2}{|c|}{$\begin{array}{c}\text { Presencia } \\
(N=253)\end{array}$}} & \multirow{2}{*}{\multicolumn{2}{|c|}{$\begin{array}{c}\text { Ausencia } \\
(\mathrm{N}=183)\end{array}$}} & \multirow[b]{2}{*}{ OR } & \multirow{2}{*}{$\begin{array}{c}\mathrm{IC} 95 \\
\%\end{array}$} & \multirow{2}{*}{$\begin{array}{l}\text { Valor } \\
\mathrm{P}^{\star}\end{array}$} \\
\hline & & & & & & & \\
\hline $\begin{array}{l}\text { Edad en años } \\
\text { (media/desviación) }\end{array}$ & \multicolumn{2}{|c|}{$(8,4 / 1,8)$} & \multicolumn{2}{|c|}{$(7,7 / 2,0)$} & & & $<0,001^{*}$ \\
\hline $5-6$ & 45 & 17,8 & 67 & 36,6 & 1,0 & & 0,002 \\
\hline $7-8$ & 81 & 32,0 & 50 & 27,3 & 2,4 & $1,4-4,0$ & \\
\hline $9-10$ & 88 & 34,8 & 47 & 25,7 & 2,8 & $1,7-4,7$ & \\
\hline $11-12$ & 39 & 15,4 & 19 & 10,4 & 3,1 & $1,6-6,0$ & \\
\hline \multicolumn{8}{|l|}{ Zona } \\
\hline Rural & 119 & 47,0 & 91 & 49,7 & 1,0 & & 0,578 \\
\hline Urbana & 134 & 53,0 & 92 & 50,3 & 1,1 & $0,8-1,6$ & \\
\hline \multicolumn{8}{|l|}{ Tipo de dentición } \\
\hline Inicial & 10 & 4,0 & 33 & 18,0 & 1,0 & & 0,000 \\
\hline $\begin{array}{l}\text { Mixta } \\
\text { intertransicional }\end{array}$ & 87 & 34,4 & 65 & 35,5 & 4,4 & $2,0-9,6$ & \\
\hline Mixta final & 78 & 30,8 & 41 & 22,4 & 6,3 & $2,8-14,0$ & \\
\hline Permanente & 57 & 22,5 & 31 & 16,9 & 6,1 & $2,6-13,9$ & \\
\hline $\begin{array}{l}\text { Dentición } \\
\text { decidua }\end{array}$ & 21 & 8,3 & 13 & 7,1 & 5,3 & $2,0-14,3$ & \\
\hline
\end{tabular}

*Prueba Chi-cuadrado (usando regresión logística); **Prueba de Mann -Whitney

Tabla 4. Características de individuos según "espaciamiento"

\begin{tabular}{|c|c|c|c|c|c|c|c|}
\hline \multirow[t]{2}{*}{ Característica } & \multicolumn{2}{|c|}{$\begin{array}{l}\text { Presencia } \\
(\mathrm{N}=253)\end{array}$} & \multicolumn{2}{|c|}{$\begin{array}{c}\text { Ausencia } \\
(\mathrm{N}=183)\end{array}$} & \multirow[t]{2}{*}{ OR } & \multirow{2}{*}{$\begin{array}{c}\mathrm{IC} 95 \\
\%\end{array}$} & \multirow[t]{2}{*}{ Valor $\mathrm{P}^{\star}$} \\
\hline & & & $n$ & & & & \\
\hline $\begin{array}{l}\text { Edad en años } \\
\text { (media/desviación) }\end{array}$ & \multicolumn{2}{|c|}{$(7,7 / 1,9)$} & \multicolumn{2}{|c|}{$(9,0 / 1,7)$} & & & $<0,001^{*}$ \\
\hline $5-6$ & 97 & 33,3 & 15 & 10,3 & 1,0 & & 0,000 \\
\hline $7-8$ & 100 & 34,4 & 31 & 21,4 & 0,5 & $0,3-1,0$ & \\
\hline $9-10$ & 61 & 21,0 & 74 & 51,0 & 0,1 & $0,1-0,2$ & \\
\hline $11-12$ & 33 & 11,3 & 25 & 17,2 & 0,2 & $0,1-0,4$ & \\
\hline \multicolumn{8}{|l|}{ Zona } \\
\hline Rural & 135 & 46,4 & 75 & 51,7 & 1,0 & & 0,293 \\
\hline Urbana & 156 & 53,6 & 70 & 48,3 & 1,2 & $0,8-1,9$ & \\
\hline \multicolumn{8}{|l|}{ Tipo de dentición } \\
\hline Inicial & 36 & 12,4 & 7 & 48 & 1,0 & & 0,000 \\
\hline $\begin{array}{l}\text { Mixta } \\
\text { intertransicional }\end{array}$ & 132 & 45,4 & 20 & 13,8 & 1,3 & $0,5-3,3$ & \\
\hline Mixta final & 67 & 23,0 & 52 & 35,9 & 0,3 & $0,1-0,6$ & \\
\hline Permanente & 42 & 14,4 & 46 & 31,7 & 0,2 & $0,1-0,4$ & \\
\hline Dentición decidua & 14 & 4,8 & 20 & 13,8 & 0,1 & $0,1-0,4$ & \\
\hline
\end{tabular}


$\mathrm{Al}$ analizar las variables en los tres planos del espacio en individuos con dentición decidua, se encontró en el plano sagital que la relación molar de plano terminal recto se acompañaba con mayor frecuencia de la relación canina clase I en un 56,8 \% (100/82), la relación molar escalón distal con una relación canina clase I en un 43,5 \% (67/82) y finalmente una relación molar escalón mesial con una relación canina clase II en un 25 \% (6/82) (Tabla 3). Con respecto al overjet se encontró adecuado en un 75,6 $\%$ (62/82), aumentado en un 9,8 \% (8/82), invertido en un 2,4 \% (2/82) y no se pudo determinar por ausencia de incisivos en un 12,2 \% (10/82). En el plano vertical se encontró un overbite adecuado del $28 \%$ (23/82), una mordida profunda en el 47,6 \% (39/82), abierta en un 1,2 \% (1/82) y una mordida borde a borde en un 9,8 \% (8/82). Finalmente en el plano trasversal se encontró una mordida cruzada completa en un 6,1 \% (5/82), presentándose en un 7,3 \% (6/82) de manera unilateral y de manera bilateral en un 2,4 \% (2/82). Con respecto a la línea media dental se observo coincidencia en un $86.6 \%$ (71/82) y no coincidencia en un 13,4 \% (11/82). Cuando se valoro el apiñamiento se observo un 30 \% (25/82) de frecuencia, presentándose en un 31,7 \% (26/82) en la zona anterior del arco, no se presento en la zona posterior, no se pudo determinar por ausencia de incisivos en un 68,3\% (56/82) y no existió apiñamiento en un 69,5 \% (57/82). De otro lado, el espaciamiento se presento en un 87,8 \% (72/82) en la zona anterior del arco en un $87,8 \%$ (72/82) y en la zona posterior no se observo espaciamiento y no se pudo determinar por ausencia de incisivos en un 12,2 \% (10/82). Cuando se valoró la existencia de diferencias en las proporciones de caracterización de la muestra en los grupos con maloclusión en dentición permanente y con maloclusión en dentición decidua por variable socio demográfica evaluada, se encontró que si existían diferencias estadísticamente significativas en edad, y tipo de dentición con un valor de $p$ menor 0,005 y un $\mathrm{Z}$ del $95 \%$ (Tabla 4).

Cuando se evaluó las características de la muestra comparando individuos con maloclusión en dentición permanente con individuos sin maloclusión buscando variables sociodemográficas que estuvieran asociadas a la maloclusión, ninguno de los datos de razón de disparidad (RD) fue mayor al valor de 2 y su correspondiente valor de $\mathrm{p}$ no fue estadísticamente significativo ( $\mathrm{p}>0.05$ ). Lo mismo ocurrió al comparar los dos grupos de maloclusión en dentición permanente y maloclusión en dentición decidua con individuos sin maloclusión, aunque el valor de p fue estadísticamente significativo (<0,05-Z $95 \%)$ al comparar la diferencia entre los 2 grupos. 
Cuando se valoró las características de la muestra de maloclusión en dentición permanente clase II y III con la maloclusión clase I/maloclusión en dentición decidua se encontró un grado de asociación para el rango de 9 a 10 años seguido del rango de 7 a 8 años. De igual forma esta asociación se presento en la dentición mixta transicional con una menor fuerza con un valor de $p$ estadísticamente significativo $(\mathrm{p}<0.05)$.

\section{DISCUSION}

Durante todo el desarrollo de la dentición es muy frecuente encontrar alteraciones en la posición de los dientes que conllevan a desarmonías oclusales. La prevalencia de las alteraciones oclusales en el plano sagital en adolescentes ha sido descrita en diferentes poblaciones, con una frecuencia de 70 \% en Estados Unidos (6), 77 \% en Venezuela (2); en Colombia, Thilander realizó un estudio en Bogotá en el 2001 en el cual identificó que la prevalencia de la maloclusión era del 88 \% en niños y adolescentes (8). Estudios regionales en Antioquia reportan una prevalencia en la ciudad de Medellín en un rango entre el 86.4 \% y 97.4 \% en niños entre los 2 a 12 años; nuestro estudio identifico una prevalencia del 94 \% de maloclusión en niños de edad escolar entre los 5 a los 12 años específicamente en Envigado.

$\mathrm{Al}$ analizar el tipo de maloclusión más frecuente en Europa se encuentra que la maloclusión más prevalente es la clase I con $79 \%$, seguido de la maloclusión clase II con un 18 \% y finalmente la clase III con un $3 \%$ (7), en Estados Unidos la prevalencia reportada es para la clase I de un 61,6\%, para la clase II de 34,3 \% seguido de la clase III con un $4.1 \%$ (6), en Latinoamérica encontraron una prevalencia del $93 \%$ para la clase I y 7 \% para la clase II y finalmente un $1 \%$ para la clase III. A nivel nacional Thilander et al. En su estudio reporta que la maloclusión clase I es la de mayor prevalencia con un 62,7 \%, seguida de la clase II con un $20.8 \%$, y la de menor prevalencia fue la maloclusión clase III con un 3.7\%(5). Pocos estudios se han realizado a nivel regional, uno de los cuales reportado por Botero y col en un centro universitario del municipio de Envigado, el cual determinó que la prevalencia de la maloclusión para la clase I fue del 55 \% seguida de la clase II $32.5 \%$ y por último la clase III con un $12.6 \%$ (2). El presente estudio reveló que la maloclusión más frecuente era la clase I con una prevalencia de $49.7 \%$, seguida de la maloclusión clase II con un 43.5 \% y 6.8 \% para la clase III donde se encuentra similitud con los otros 
estudios, aunque las maloclusiones II y III se muestran más prevalentes en la población estudiada. En el plano transversal la prevalencia de las mordidas cruzadas del 11 \% reportada en este estudio es mayor a la de Europa con el 7,7 \% (7), el de Venezuela con el 6,5\%(8), Bogotá con el 3,5 \% (5), similar a la de Medellín con el $11 \%(4)$ y menor prevalencia a la encontrada en Brasil con el 20.8 \%(9). Sin embargo se debe tener en cuenta que los niños evaluados en Brasil se encontraban entre los 3 y lo 6 años únicamente (9).

Con respecto al overbite se encuentra que la mordida profunda es la más común de las alteraciones verticales con una prevalencia de 39,2 \% para este estudio y entre 13,6 \% (4) y 46,2 \% (9) en los estudios reportados en el ámbito nacional como internacional (2-7.9-11). La mordida abierta en general se presenta con una menor prevalencia entre 2,8 \% y el 17,8 $\%$ incluyendo este estudio donde la prevalencia fue baja con un $3 \%$. En nuestro estudio el apiñamiento se encuentra en una alta prevalencia del $57,8 \%$ con una ubicación anterior del 58 \% similar a lo reportado en otros estudios (4, 8-14). Al comparar la dentición decidua con la permanente en nuestro estudio se encuentra que la mordida profunda es mayor en deciduos (47,6 \%) que en permanentes (39,8\%), la mordida cruzada mayor en permanentes (11 \%) que en deciduos ( $8,5 \%$ ) y el apiñamiento es mayor en permanentes con un 57,8 \% contra un $30 \%$ en los deciduos. Sin embargo es importante resaltar que los individuos en dentición decidua eran más pocos que los individuos en dentición permanente

Agradecimientos: Financiado por el CONADI "Comité Nacional para el desarrollo de la Investigación", de la Universidad Cooperativa de Colombia. Instituciones educativas que brindaron apoyo para la realización del proyecto: Institución educativa José Manual Restrepo, Escuela San Rafael, Escuela Santo Domingo Savio, Institución educativa el salado, Centro Educativo rural la cruz del porvenir, C.E.R Martín Eduardo Ríos Llanos, Centro Educativo rural la morena, Centro educativo loma José Dolores, Institución educativa las palmas, Centro educativo rural alto de las flores, Politécnico Integral Colombiano, Colegio Integrado Laureles, Colegio de Capacitación de Envigado, Indecap, institución Educativa Nocturno Ayurá. 


\section{REFERENCIAS}

1. Brusola J. Ortodoncia Clínica y terapéutica. 2da Edicion. España: MASSON; 2000.

2. Onyeaso CO. Prevalence of malocclusion among adolescent in Ibadan Nigeria. Am J Orthod Dentofacial Orthop 2004; 126:605-607.

3. Isper AJ, Pantaleão dos Santos MR, Gonçalves PE. Prevalencia de maloclusión en la dentición primaria en el municipio de Cáceres Brasil. Rev Cubana Estomatol 2007; 45(1):91-95.

4. Botero PM, Vélez N, Cuesta DP, Gómez E, Cossio M, Santos E. profile of dental occlusion in children from Universidad Epidemiological Cooperativa de Colombia. Rev CES Odont 2009; 22(1): 9-13.

5. Ministerio de salud de Colombia. III Estudio Nacional de Salud Bucal (ENSAB III) Bogota. Ministerio de salud 2004:85-147.

6. Thilander B. Prevalence of malocclusion and orthodonctic treatment need in children and adolescents in Bogota Colombia. An epidemiological study related to different stages of dental development. European Journal of Orthodontics 2001; 23:153167.

7. Álvarez EF, Roldán S, Mejía J, Tobón 6. P, Velásquez L. Estudio de la prevalencia, distribución y severidad de la maloclusión de la población escolar de 7 a 16 años de la ciudad de Medellín. Academia Colombiana de Odontología Pediátrica 2002; 4(1):25-34.

8. Bishara SE. Changes in the molar relationship betweenthe deciduous and permanent dentitions:A longitudinal study. Am J Orthod Dentofacial Orthop1988; 93:19- 28.

9. Tausche OL. Prevalence of malocclusions in the early mixed dentition and orthodontic treatment need. Eur J Orthod 2004; 26:237-244.

10. Cano C, Gutiérrez N, Velásquez Y,Godoy S, Quiros O, Farias M. Frecuencia de la maloclusion en niños de 5 a 9 años en una zona rural del estado de Guarico periodo 2007 - 2008. Revista Latinoamericana de ortodoncia y odontopediatría [internet]. Disponible en: http://www.ortodoncia.ws/publicaciones/2008/frecuencia_ maloclusion ninos_estado_guarico.asp

11. Abreu EB. Prevalência de má-oclusão e fatores relacionado à sua ocorrência em préescolares da cidade de São Luís - MA - Brasil. RPG Rev Pós Grad 2005; 12(2):212221.

12. Scott JH. The shape of dental arches. J Dent Res 1957; 36:996-1003.

13. Moyers RE, Van der Linden P, Riolo ML et al. Standars of Human Occlusal Development, Monoghraph 5, Craniofacial Growth series. Ann Arbor Mich. Center for Human Growth and Development, University 1976.

14. Hunter WS, Smith BR. Development of mandibular spacing-crowding from nine to sixteen years old of age. J Can Dent Assoc 1972; 38:178-185.

15. Baume LJ. Phisiologycal Tooth migration and its significance for the development of occlusion. J Dent Res 1950; 29(2):123-132. 